\title{
An experimental study of fluid dynamics of stall delay on the blade of a horizontal-axis wind turbine*
}

\author{
[Yanhua Wu, Shaoqiong Yang, Zhanqi Tang]
}

\begin{abstract}
Fluid dynamics of the stall delay phenomenon is studied experimentally in this work on a horizontal-axis wind turbine (HAWT) blade. The 3D-printed blade is produced from a downscaled model of a $5 \mathrm{~kW}$ HAWT. Its rotational motion in the test section of wind tunnel is precisely controlled by a programmable motor. Both mean static pressures and dynamic pressures are measured on the suction surface at the spanwise location of $0.25 \mathrm{R}$ of the blade at two different global tip speed ratios (TSR) of 3 and 5. The TSR of 3 corresponds to a large angel of attack (AOA) at 30 degrees where the flow is fully separated. The other TSR of 5 corresponds to a smaller AOA of 15 degrees. Planar time-resolved particle image velocimetry measurements at $0.25 \mathrm{R}$ for the above two TSRs were performed to study the dynamics of the separated flows when the stall is delayed on the rotating blade. The Reynolds numbers at these two TSRs, based on the half chord length of the blade and its relative velocity, are 8683 and 6695 , respectively. Finally, the possible correlation between the dynamics of pressure and the time evolution of shed vortices is analyzed during stall delay.
\end{abstract}

Keywords - Stall delay, horizontal-axis wind turbine, static pressure, dynamic pressure, time-resloved particle image velocimetery

\section{Introduction}

In fluid dynamics, a stall is a reduction in the lift coefficient generated by a foil as angle of attack increases ${ }^{[1]}$. The stall delay phenomenon is a 72-year-old mystery already which was observed for a first time by a technician, Himmelskamp ${ }^{[2]}$ who found that the lift coefficients at the inboard section (i.e. close to the root of blade) of a rotating blade were significantly higher than the maximum value possibly obtained in a two-dimensional (2D) static airfoil in 1945. The stall delay is well known in many engineering applications involving turbo-machineries such as wind, steam and gas turbines, helicopter propellers, and diffusers, etc. Besides, in physics and fluid mechanics, a boundary layer

Yanhua Wu, Shaoqiong Yang, Zhanqi Tang

Nanyang Technological University

Singapore

Shaoqiong Yang, Zhanqi Tang

Tianjin University

China

* This work is financially supported by Ministry of Education in Singapore under the Project No. RG 52/11.

refers to the layer of fluid in the immediate vicinity of a bounding surface where the effects of viscosity are significant ${ }^{[3]}$. Therefore, The stall delay herein also means that the flow separation of boundary layer is delayed on the rotating blades. The phenomenon for stall delay was then found and studied by Wood and his co-workers ${ }^{[4,5]}$ on the wind turbine blades. However, The stall delay remains one of the unknown sciences so far to human beings.

From 1991, Wood ${ }^{[6]}$ started to investigate the fluid physics of stall delay. He performed a three-dimensional (3D) panel analysis of a horizontal-axis wind turbine and found that when the stall approached, the large negative pressure gradients near the leading edge of a rotating airfoil were reduced. Therefore, he concluded that the delayed boundary layer separation on the suction surface was as a result of this adverse pressure gradient reduction. However, Dumitrescu and Cardos ${ }^{[7]}$ argued that the boundary layer separation delayed by the effects of rotation was due to the Coriolis force based on their numerical results. In other numerical simulation studies, Chaviaropoulos and Hansen $^{[8]}$ revealed that when flow was massively separated at high angle of attack (AOA) [The AOA herein is the angle between the chord line of airfoil and the oncoming flow.], the Coriolis force redirected the flow within the separation bubble to the radial direction and thus reduced the volume of separated flow which in turn induced a pressure drop on the suction surface of the rotating blade. Hu et al. ${ }^{[9]}$ compared the flow structures and pressure coefficients of the static airfoil and rotating blade at similar AOAs and Reynolds number $(R e)$ [The Reynolds number, in fluid dynamics, is the ratio of inertial forces to viscous forces within a fluid which is subjected to relative internal movement due to different fluid velocities.], which concluded that the stall was delayed on the rotating blade due to the chordwise pressure gradient produced by the Coriolis forces which were against the adverse pressure gradient along the blade chord. Du and Selig ${ }^{[10]}$ believed that Coriolis forces accelerated the flow in the chordwise direction toward the trailing edge and resulted in the reduction of the adverse pressure gradient, which delayed the occurrence of separation to a point further downstream. Yang et al. ${ }^{[11]}$ found that the lift losses were strongly reduced in post-stall conditions by rotation at the inboard sections. They concluded that the main effects of rotation were to stabilize vortex shedding and to limit the growth of separation. Dumitrescu et al. $^{[12]}$ simulated the 3D streamlines on the suction surface of the blade designed by NREL and they claimed that the centrifugal forces produced a center-wise suction effect which stabilized the root vortex located above the suction surface of blade. This vorticity concentration process near the root region postponed the separation and resulted in lift augmentation.

From the year of 2012, Wu and his co-workers ${ }^{[13]}$ started to experimentally study the physics of stall delay on the blade of a $5 \mathrm{Kw}$ horizontal-axis wind turbine by Particle Image Velocity (PIV). They, for the first time, measured the detailed threedimensional and volumetric velocity fields using Tomographic PIV on a model of the rotating blade of a small-scale HAWT 
to study the physics of stall delay at two different global tip speed ratios (TSRs) [The tip-speed ratio or TSR for wind turbines is the ratio between the tangential speed of the tip of a blade and the actual speed of the wind.] at about $R e=5000^{[14-18]}$. Their results showed that rather than the recirculation separation bubbles with strong reversed flows for the static airfoil at deep stall, the attached flows were observed on the suction surface of the rotating blade ${ }^{[14,15,17]}$. Radial flows from the root of blade to tip were also found with strong spanwise velocity component, located in the vicinities of the vortices and close to the suction surface of blade. In contrast to the airfoil case, the vortices shed from the edges of blade to break down into smaller ones were not found ${ }^{[14,17]}$. The surface streamlines of blade revealed complex 3D flows close to the suction surface of the blade. They also found that Coriolis forces were larger than centrifugal forces in chordwise direction in measurement volumes from inboard to outboard at large AOAs, which contributes to the reduction of the adverse pressure gradient ${ }^{[14]}$. It, therefore, delayed the stall. In addition, they observed that although the frees-stream turbulence levels affected the separated flows for the static airfoil, they have much less effects on the rotating blade ${ }^{[16]}$. Furthermore, in the near wake region of a airfoil in deep stall, the turbulence structures involved the large-scale Karman vortex and interactions with the small-scale shear layer vortices ${ }^{[18]}$.

Based on the above results, It is still not to fully disclose the fluid physics of stall delay so far. Especially, the dynamics of the flow structures and the turbulence generated by the rotating blade when the stall delay. Besides, we need to recover the important parameters, such as TSR and Reynolds number effects on the dynamics of these structures. For this, in present study, Both mean static pressures and dynamic pressures were measured on the suction surface at the spanwise location of $0.25 \mathrm{R}$ of the blade at two different TSR of 3 and 5. Planar time-resolved PIV measurements at $0.25 \mathrm{R}$ for the above two TSRs were performed to study the dynamics of the separated flows when the stall is delayed on the rotating blade with the corresponding Reynolds number of 8683 and 6695 , respectively.

\section{Experimental setups}

\section{A. Flow facility and blade model}

Experiments were performed in two low-turbulence, open circuit, Eiffel type wind tunnels with a test section (Width $\times$ Height $\times$ Length) of $0.4 \mathrm{~m} \times 0.4 \mathrm{~m} \times 2.4 \mathrm{~m}$ (Hereinafter referred to as small wind tunnel) and $1.1 \mathrm{~m} \times 0.9 \mathrm{~m} \times 2 \mathrm{~m}$ (Hereinafter referred to as large wind tunnel), respectively. The free-stream turbulence intensity in the test sections were both less than $1 \%$. The side walls of the small wind tunnel are made of glass and the other walls of the two wind tunnel are made of acrylic for optical access. The wind turbine blade is 3D-printed and it produced from a downscaled model of a $5 \mathrm{~kW}$ horizontal-axis wind turbine (HAWT), whose entire radius ( $\mathrm{R}$ ) is about $0.42 \mathrm{~m}$ (See Fig. 1 (a)). Its rotational motion in the test section of wind tunnel is precisely controlled by a programmable motor. The design TSR of the rotor blade is 6 . The TSR is defined as

$$
\mathrm{TSR}=\frac{\Omega \mathrm{R}}{\mathrm{V}}
$$

where $\Omega$ is the rotating speed of the wind turbine blade and $V$ is the free-stream velocity.

The entire downscale blade has been cut-off to $0.185 \mathrm{~m}$ for planar time-resolved PIV measurements in the small wind tunnel (See Fig.1 (c)).

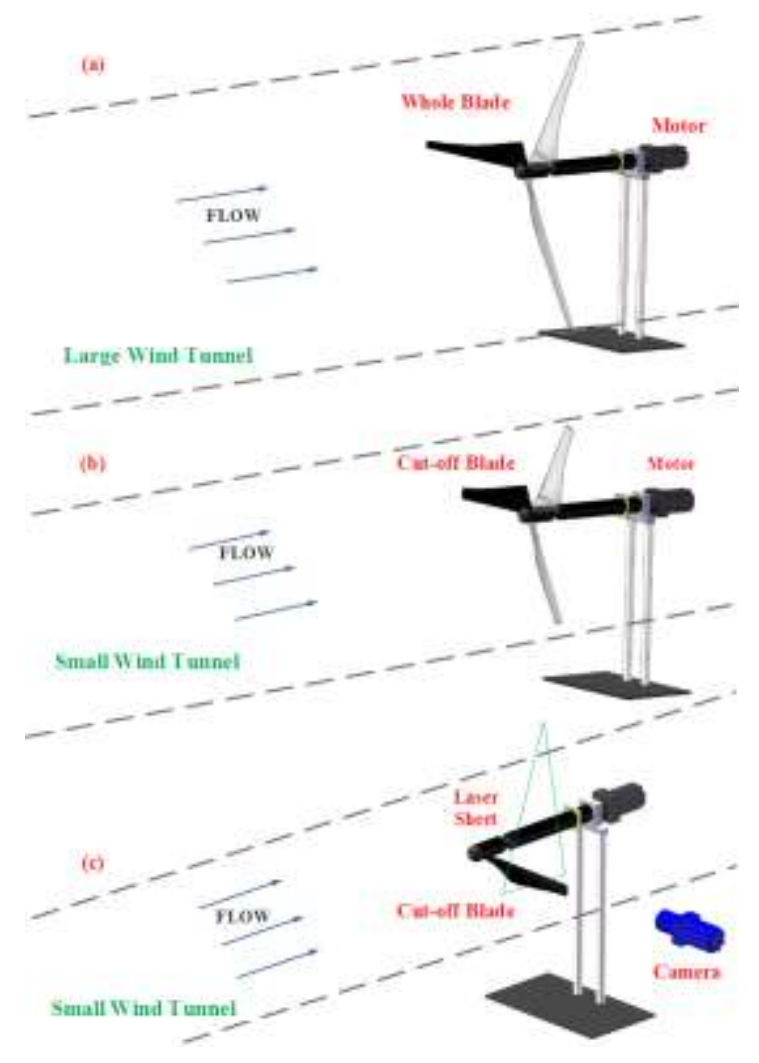

Figure 1. The diagram of experimental setup. The pressure measurements inside the small (a) and large (b) wind tunnel according to the different chord length of the blade of wind turbine (The plastic tubes are not shown for clarity); and (c) PIV measurements inside the small wind tunnel for the cut-off blade.

The experiments were conducted out at two different freestream velocities, which then yield two different global TSR of 3 and 5. The TSR of 3 corresponds to a large AOA at 30 degrees where the flow is fully separated. The other TSR of 5 corresponds to a smaller AOA of 15 degrees. Both mean static pressures and dynamic pressures as well as 2D PIV measurements are measured on the suction surface at the spanwise location of $0.25 \mathrm{R}$ of the blade for corresponding two different Reynolds numbers, 8683 and 6695, respectively. Reynolds number herein is based on the half chord length of the blade, $c_{m}$, and the relative velocity, $V_{r}$, and the kinematic viscosity of air, $\mathrm{v}$, i.e. $R e=V_{r} c_{m} / \mathrm{v}$.

The relative velocity, $V_{r}$, at $0.25 \mathrm{R}$ is calculated by

$$
V_{R 0.25}=\sqrt{V_{\infty}^{2}(1-a)^{2}+\left(\Omega R_{0.25}\right)^{2}\left(1+a^{I}\right)^{2}},
$$


where $a$ and $a^{\prime}$ are axial and angular induction factors, respectively, obtained from popular Blade Element Momentum (BEM) method (See Reference ${ }^{[19]}$ and its Appendix A) during the design of the wind turbine blade.

The AOA can then be determined by

$$
\mathrm{AOA}=\gamma-\beta
$$

Where $\gamma$ is the angle between the free-stream velocity and chord; and $\beta$ is the angle between the free-stream and relative velocity.

$\beta$ can be estimated by

$\tan \quad=\quad \frac{\lambda_{r}\left(1+a^{\prime}\right)}{1-a^{\prime}}$

where is local TSR.

\section{B. Pressure measurements}

Figure 2 shows 14 pressure taps on the suction surface of the blade. Chordwise positions for the static pressure tapping hole, $x_{14}=18.3 \times 0.135 \mathrm{~mm}$, and then $25 \times 0.135 \mathrm{~mm}$ increment for each tap (See Table 1). The static pressure on the suction surface were measured to obtain information of the pressure distribution along the whole suction surface when the blade rotates inside the small and large wind tunnel, respectively. Note that pressure measurements cannot be performed at near the leading and trailing edges due to its low thickness and small chord at the tip region of the blade. The fourteen pressure taps were connected by plastic tubes to a differential pressure transducer whose model number is OMEGA PX163-2.513135V. The measurement range of the differential pressure sensor is $\pm 622.1 \mathrm{~Pa}$ and its accuracy within calibration is about $1 \%$ of the full scale.

The pressure coefficient $\left(C_{p}\right)$ of the rotating blade was obtain by

$$
C_{p}=\frac{\mathrm{P}_{\mathrm{ro}}-\mathrm{P}_{\infty}}{0.5 \times \rho V_{\mathrm{r}_{0.25}}^{2}}
$$

where $\mathrm{P}_{\text {ro }}$ is the static pressure measured on the rotating blade, $\mathrm{P}_{\infty}$ is the free-stream static pressure far upstream from the rotating blade and $\rho$ is the density of air.

A dynamic pressure measurement system that includes a microphone sensor (Brüel \& Kjær, Surface Microphone Type 4949), data acquisition (NI USB-6000) and the conditioning unit was used to measure the dynamic pressure on the suction surface of the rotating blade near the tenth tap hole at the spanwise location of $0.25 \mathrm{R}$. The diameter of diaphragm of the microphone is $10.3 \mathrm{~mm}$. Its sensitivity is $11.2 \mathrm{mV} / \mathrm{Pa}$ and frequency range $( \pm 3 \mathrm{~dB})$ is from 5 to $20 \mathrm{kHz}$.

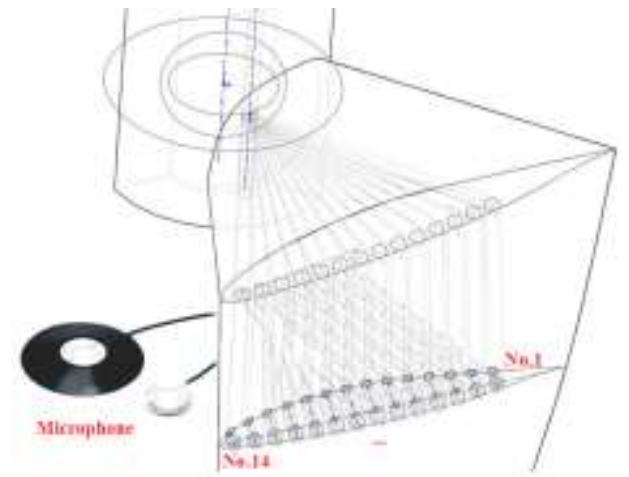

Figure 2. The stastic pressure tap locations showing how to be connected with the plastic tubes inside the blade and the insets are the surface microphones (Type 4949) using for dynamic pressure measurements.

\begin{tabular}{|c|c|c|c|c|c|c|c|c|c|c|c|c|c|}
\hline $\begin{array}{c}\text { Leading } \\
\text { Edge }\end{array}$ & \multicolumn{2}{|c|}{ No.14 } & \multicolumn{2}{|c|}{13} & \multicolumn{2}{|c|}{12} & \multicolumn{2}{|c|}{11} & \multicolumn{2}{|c|}{10} & \multicolumn{2}{|c|}{9} & 8 \\
\hline$x / c_{0.25}=0$ & \multicolumn{2}{|c|}{0.04} & \multicolumn{2}{|c|}{0.1} & \multicolumn{2}{|c|}{0.16} & & & \multicolumn{2}{|c|}{0.28} & \multicolumn{2}{|c|}{0.33} & 0.39 \\
\hline 7 & 6 & & & & 4 & & & & & & & & $\begin{array}{l}\text { iling } \\
\text { lge }\end{array}$ \\
\hline 0.45 & 0.51 & & & & 62 & & & & & & & & ${ }_{25}=1$ \\
\hline
\end{tabular}

TABLE I. PRESSURE TAP LOCATIONS

\section{Time-resolved PIV measurements}

Figure 1(c) shows the schematic of the experimental setup for the time-resolved PIV measurements. Seeding particles with a nominal diameter of about $1 \mu \mathrm{m}$, which were generated by a Laskin nozzle, were illuminated by a thin laser sheet. The laser sheet less than $1 \mathrm{~mm}$ thick was produced by a pair of $140 \mathrm{~mJ} /$ pulse Nd: YAG PIV lasers at the spanwise location of $0.25 \mathrm{R}$. The particles images were recorded at $2000 \mathrm{~Hz}$ by a 12-bit high speed camera (LaVision, FASTCAM Mini UX100) with a $60 \mathrm{~mm}$ lens which was configured perpendicularly to the laser sheet. The blade rotates in the test section of the small wind tunnel and the trigger of the camera is phaselocked, through the usage of a laser sensor (Keyence, LVN11P), to the horizontal position of the blade when the blade is rotating upward controlled by the programmable motor, which is shown in Fig 1(c).

A total of more than 1200 sets of images with each set of 15 image pairs after the camera being triggered were captured for studying the dynamics of the flow behind the suction surface of the rotating blade when the stall delays. The planar velocity vector fields were calculated by $\mathrm{DaVis}$ 8.3.0 with multi-pass iterations when the window sizes are $48 \times 48$ and $24 \times 24$ with the overlap is $50 \%$. At last, the vector postprocessing are used to finally obtain $107 \times 86$ vectors for each flow field. The estimation on the PIV uncertainties for the velocities measurements can found in $\mathrm{Wu}$ and Christensen ${ }^{[20]}$. The random uncertainty for the present study is about $0.3 \%$ and the bias uncertainty is about $3 \%$. 


\section{Results and discussions}

\section{A. Static pressures}

Figure 3 shows the static pressure coefficients, $-C_{p}$ distributions along the chordwise direction on the suction surface at the spanwise location of $0.25 \mathrm{R}$ of the rotating blades when TSRs are 3 and 5, respectively. The Reynolds numbers are 8683 and 6695 for the present study. Besides, as described in Section II, Static pressure data for the entire blade rotated inside large wind tunnel (LWT) and the cut-off blade rotated inside small wind tunnel (SWT) are together shown in the figures for comparison. And the results of Lee ${ }^{[19]}$ at the same TSRs and similar AOAs, but different Reynolds numbers are drawn in as well. $-C_{p}$ of these rotating blades at $0.25 \mathrm{R}$ along the chordwise show higher values than the static airfoil ${ }^{[14,15]}$ at the same AOAs either TSR=3 or TSR=5, which is the similar to Lee's results. Meanwhile, within the error range, the mean static pressures across the chord at $0.25 \mathrm{R}$ are almost the same, no matter for the entire blade or for the cut-off one. That means the averaged static pressure distributions are not affected by the cutting when the Reynolds number and TSR are the same, at least for the spanwise location at $0.25 \mathrm{R}$ of the blade.
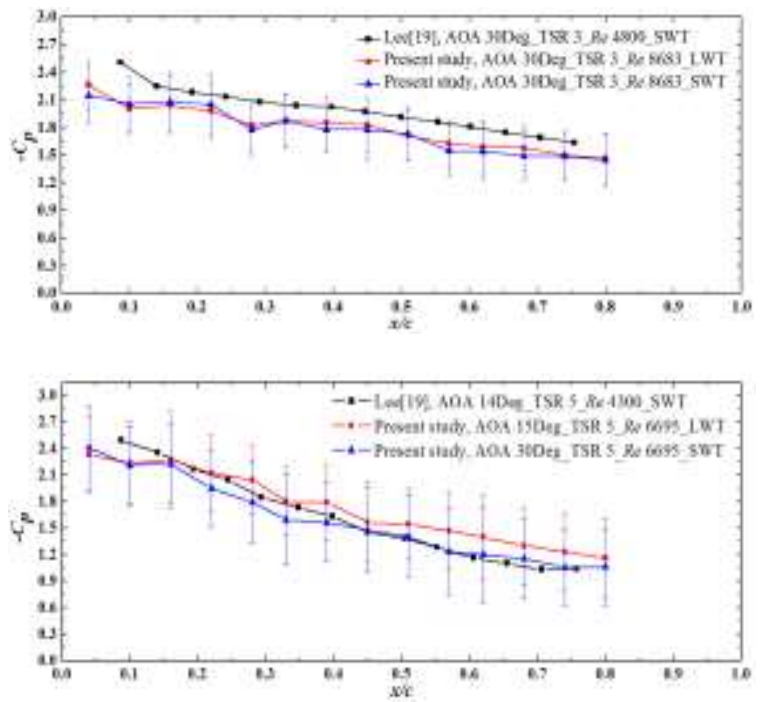

Figure 3. The stastic pressure coefficients' distributions along the chordwise direction at the spanwise location of $0.25 \mathrm{R}$ of the rotating blade at TSR $=3$ (up) and $\mathrm{TSR}=5$ (down).

\section{B. Dynamic pressures}

The dynamic pressures on the suction surface of the rotating blade near the tenth tap at the spanwise location of $0.25 \mathrm{R}$ were measured by the surface microphone at a sampling frequency of $750 \mathrm{~Hz}$. The original signals were filtered with the cut-off frequency from 1 to $50 \mathrm{~Hz}$; and then a Fast Fourier Transform (FFT) was performed to obtain the frequency domain information about the dynamic pressures as shown in Fig.4. The frequency peaks at $3.5 \mathrm{~Hz}$ are the same for the two TSRs of 3 and 5, which is much lower than the vortex shedding frequency for the static airfoil at the corresponding AOAs of 30 or 15 degrees indicating that the stall was delayed.

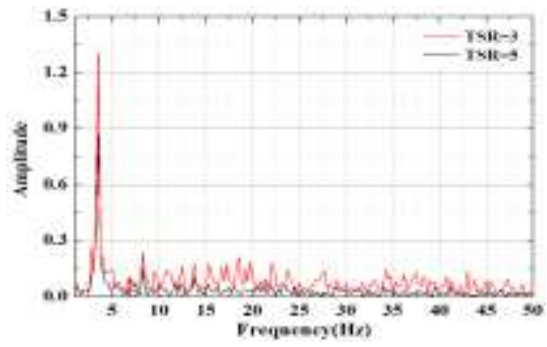

Figure 4. The dynamic pressure on the suction surface of the rotating blade near the tenth tap hole at the spanwise location of $0.25 R$ when $T S R=3$ and 5 .

\section{Time-resolved PIV results}

The instantaneous velocity snapshots to the rotating blade at the spanwise location, $0.25 \mathrm{R}$, of the rotating blade at TSR $=3$ and TSR=5 are shown in Fig.5, respectively. The iso-contours represent the vorticity. There is a large vortex behind the rotating blade and the flow started to partially separate, which is different with the case for the static airfoil at the AOA of 30 degree ${ }^{[14,16]}$ where the flow is fully separated; while the flow at TSR=5 is still attached, which is the same as the previous results ${ }^{[14]}$. Note that a strong reversed flow from the trailing edge to leading edge at near the suction surface of the blade at a higher Reynolds number $(\operatorname{Re}=8683)$ has been observed at $\mathrm{TSR}=3$, which perhaps shows the Reynolds number effects on the stall delay.
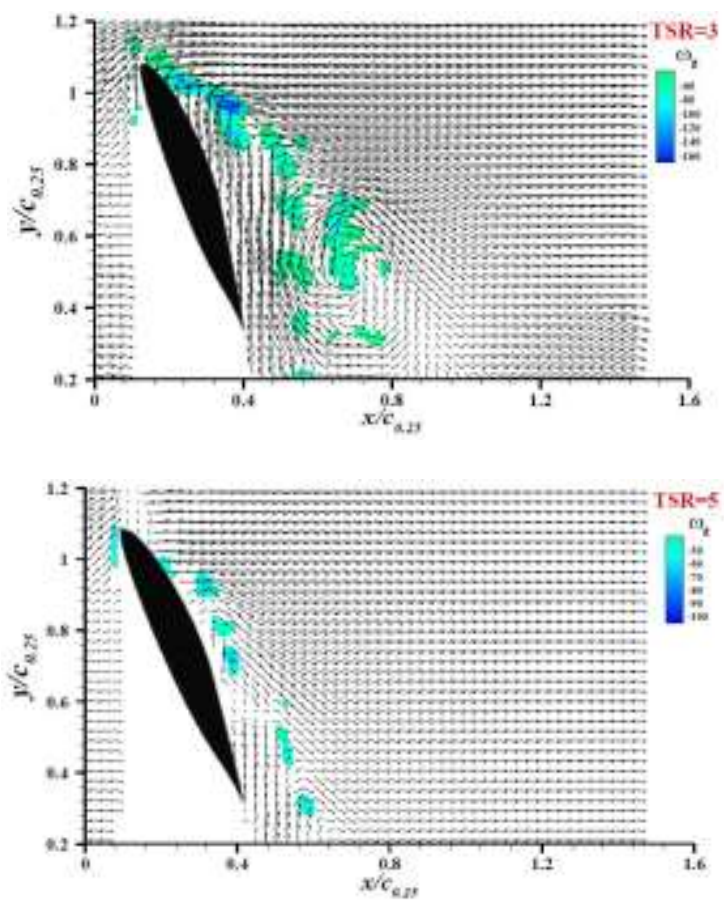

Figure 5. The instantanious velocity snapshots and vorticity contours to the rotating blade at the spanwise location, $0.25 \mathrm{R}$, of the rotating blade at TSR $=3$ and $T S R=5$. The vectors illustrate streamwise and normal velocity components. The Not every velocity vector is shown for clarity. 


\section{Conclusions}

Fluid dynamics of the stall delay phenomenon has been studied experimentally on a 3D-printed downscale HAWT blade. Both mean static pressures and dynamic pressures have been measured on the suction surface at the spanwise location of $0.25 \mathrm{R}$ of the blade at TSR is $3(R e=8683)$ and $5(R e=6695)$. Static pressure measurements' results show that $-C_{p}$ of these rotating blade at $0.25 \mathrm{R}$ along the chordwise are higher than the values for the static airfoil at the same AOAs and TSR, which is the similar to Lee's results. Besides, within the error range, the static pressures across the chord at $0.25 \mathrm{R}$ are nearly the same, either for the entire blade at the LWT or for the cut-off one at SWT. That means the mean static pressure distributions are not affected by the cutting as long as the Reynolds number and TSR are the same, at least for the spanwise location at $0.25 \mathrm{R}$ of the blade. The frequency peaks at $3.5 \mathrm{~Hz}$ for the measured dynamic pressure signals are the same when the TSR is 3 or 5 , which is much lower than the vortex shedding frequency for the static airfoil at the corresponding AOAs. Planar time-resolved PIV measurements at $0.25 \mathrm{R}$ for the above two TSRs were performed to study the dynamics of the separated flows when the stall is delayed on the rotating blade. Instantaneous velocity vector fields' results show that there is a large vortex behind the rotating blade and the flow is partially separated, which is different with the case for the static airfoil at the AOA of 30 degrees where the flow is fully separated at TSR $=3$, whose Reynolds number is 8683 . Furthermore, a strong reversed flow from the trailing edge to leading edge at near the suction surface of the blade at this Reynolds number has been observed, which perhaps shows the Reynolds number effects on the stall delay.

\section{Acknowledgment}

The authors gratefully acknowledgment the insightful comments of the reviewers; and Y. S. Q. and T. Z. Q. would like to thank Mr. Eric Yap, Lam Kim Kheong and Tan Chi Wei for their valuable experiences and precious suggestions on the experimental measurements in the wind tunnel at Nanyang Technological University, Singapore.

\section{References}

[1] Nelson H, Dictionary of Aeronautical Terms: Aviation Supplies \& Academics, Inc.,cc1991., 2012

[2] Himmelskamp H, Profile Investigations On a Rotating Airscrew, PhD Thesis, Gottingen University, 1945.

[3] Prandtl L, Zur Berechnung Der Grenzschichten, Zamm - Journal of Applied Mathematics \& Mechanics, 1938, 18: 77-82.

[4] Clausen P, Wood D, Clausen Pet al., An Experimental Investigation of Blade Element Theory for Wind Turbines. Part 2. Phase-Locked Averaged Results, Journal of Wind Engineering \& Industrial Aerodynamics, 1988, 31 (2-3): 305-22.

[5] Clausen P D, Piddington D M, Wood D H, An Experimental Investigation of Blade Element Theory for Wind Turbines. Part 1. Mean Flow Results, Journal of Wind Engineering \& Industrial Aerodynamics, 1987, 25 (2): 189-206.

[6] Wood D H, A Three-Dimensional Analysis of Stall-Delay On a Horizontal-Axis Wind Turbine, Journal of Wind Engineering \& Industrial Aerodynamics, 1991, 37 (1): 1-14.
[7] Dumitrescu H C V, Rotational Effects On the Boundary-Layer Flow in Wind Turbines, AIAA Journal, 2004, (42): 408-11.

[8] Chaviaropoulos P K, Hansen M O L, Investigating Three-Dimensional and Rotational Effects On Wind Turbine Blades by Means of a Quasi3D Navier-Stokes Solver, Journal of Fluids Engineering, 2000, 122 (2): 3306.

[9] Hu D, Hua O, Du Z, A Study On Stall-Delay for Horizontal Axis Wind Turbine, RENEW ENERG, 2014, 31 (6): 821-36.

[10] Du Z, Selig M S, The Effect of Rotation On the Boundary Layer of a Wind Turbine Blade, RENEW ENERG, 2000, 20 (2): 167-81.

[11] Yang R, Li R N, Hang W et al., Research of the Rotational Effects On the Boundary Layer of Wind Turbine Blades, MOD PHYS LETT B, 2009, 23 (03): 505-8.

[12] Dumitrescu H, Cardoş V, Inboard Boundary Layer State On Wind Turbine Blades, ZAMM - Journal of Applied Mathematics and Mechanics / Zeitschrift für Angewandte Mathematik und Mechanik, 2010, 89 (3): 163-73.

[13] Wu Y H, Lee H M, Tomographic Particle Image Velocimetry Study of Stall-Delay On the Blade of a $5 \mathrm{Kw}$ Horizontal-Axis Wind Turbine, Launceston, Australia, 2012,

[14] Lee H M, Wu Y, An Experimental Study of Stall Delay On the Blade of a Horizontal-Axis Wind Turbine Using Tomographic Particle Image Velocimetry, J WIND ENG IND AEROD, 2013, 123: 56-68.

[15] Lee H M, Wu Y, Experimental Study of Rotational Effect On Stalling, CHINESE PHYS LETT, 2013, 30 (6): 64703.

[16] Lee H M, Wu Y, A Tomo-Piv Study of the Effects of Freestream Turbulence On Stall Delay of the Blade of a Horizontal-Axis Wind Turbine, WIND ENERGY, 2014,

[17] Wu Y, Lee H M, Tang H et al., An Experimental Study of the Rotational Effects On Separated Turbulent Flow During Stall Delay, Flow, Turbulence and Combustion, 2016: 1-20.

[18] Wu Y, Tang Z, Yang S et al., Proper-Orthogonal-Decomposition Study of Turbulent Near Wake of S805 Airfoil in Deep Stall, AIAA J, 2017, 55 (6): pp. 1959-1969.

[19] Lee H M, Experimental Studies of Stall Delay On the Blade of Horizontal Axis Wind Turbine, PhD, NTU, 2013.

[20] Wu Y, Christensen K T, Reynolds-Stress Enhancement Associated with a Short Fetch of Roughness in Wall Turbulence, AIAA J, 2006, 44 (12): 3098-106.

About Author (s):

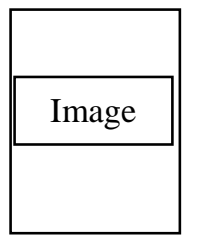

[Type a quote from the document or the summary of an interesting point. You can position the text box anywhere in the document. Use the Drawing Tools tab to change the formatting of the pull quote 